\title{
Photo-Induced Relaxation of Magnetization in Molecular Magnet
}

\author{
P.M. Zieliński ${ }^{a}$, P. Tracz ${ }^{a}$, R. Podgajny ${ }^{b}$, R. Pelka ${ }^{a}$, \\ M. BAŁAndA ${ }^{a, c}$, T. WAsiutyński ${ }^{a}$ AND B. SieKLUCKA ${ }^{b}$ \\ ${ }^{a}$ Institute of Nuclear Physics, Polish Academy of Sciences \\ Radzikowskiego 152, 31-342 Kraków, Poland \\ ${ }^{b}$ Faculty of Chemistry, Jagiellonian University \\ Ingardena 3, 30-060 Kraków, Poland \\ ${ }^{c}$ State Higher Vocational School, 33-300 Nowy Sạcz, Poland \\ Dedicated to the memory of Professor Jerzy Prochorow
}

\begin{abstract}
The experimental study of photo-induced magnetization of hybrid molecular magnet containing cobalt(II) and tungsten(V) magnetic centers bridged by $4,4^{\prime}$-bpy and $\mathrm{CN}^{-}$is presented. The observed increase in magnetization rate is attributed to the defects due to inter-valence transfer between $\mathrm{Co}^{\mathrm{II}} \mathrm{W}^{\mathrm{V}} \rightleftharpoons \mathrm{Co}^{\mathrm{III}} \mathrm{W}^{\mathrm{IV}}$. The time evolution of magnetization is parameterized by the power law rather than exponential function.
\end{abstract}

PACS numbers: 75.50.Xx, 75.60.-d, 78.20.Ls

\section{Introduction}

Since the discovery of ferromagnetic ordering in pure organic compound [1] the interest in molecular magnets is rapidly growing. Many of the affords were devoted to molecular compounds consisting of $d$-electron metallic magnetic centers. During the last decade number of spectacular achievements were reported: room temperature magnets [2], single molecule magnets [3], and photo-induced magnetism. The later one reported for the first time by Sato et al. [4] for the member of prussian blue family $\mathrm{Co}-\mathrm{Fe}(\mathrm{CN})_{6}$ triggered extensive studies of the above phenomena. As the results new materials were synthesized in which photoinduced magnetization was observed after irradiation of the sample by the light of the wavelength corresponding to the metal to metal charge transfer. It was possible to make the process reversible by applying another wavelength. The other interesting phenomena is reversing the direction of the magnetic moment by light. 
More details can be found in review paper by Hashimoto and Ohkoshi [5]. Theory of the above described phenomena based on metal to metal charge transfer was formulated by Nishino et al. [6]. The most effective process takes place when the two metal centers are connected by cyano bridge: $\mathrm{M}-\mathrm{CN}-\mathrm{M}^{\prime}$. While the first studies were performed on $3 d$ ions coordinated by six cyano ligands we extend our study to $4 d$ and $5 d$ ions: molybdenum(V) and tungsten(V) together with $3 d$ ions $(\mathrm{Cu}(\mathrm{II}), \mathrm{Ni}(\mathrm{II}), \mathrm{Co}(\mathrm{II}), \mathrm{Mn}(\mathrm{II}))$. In the present study we report the results of photo-induced magnetization on new $\mathrm{Co}_{3}^{\mathrm{II}}\left(\mathrm{H}_{2} \mathrm{O}\right)_{4}\left(4,4^{\prime} \text {-bpy }\right)_{3}\left[\mathrm{~W}^{\mathrm{V}}(\mathrm{CN})_{8}\right]_{2} \cdot 1.5\left(4,4^{\prime}\right.$ -bpy) $\cdot 6 \mathrm{H}_{2} \mathrm{O}$ (where $4,4^{\prime}$-bpy $=4,4^{\prime}$-bipyridine) coordination network which shows glass-like magnetic phase transition below $T_{\mathrm{G}}=16 \mathrm{~K}$. Every tungsten atom is coordinated by eight $\mathrm{CN}$ groups, three of them bridged to cobalt and remaining five unbridged. Cobalt atom is coordinated by two NC groups bridging to tungsten,

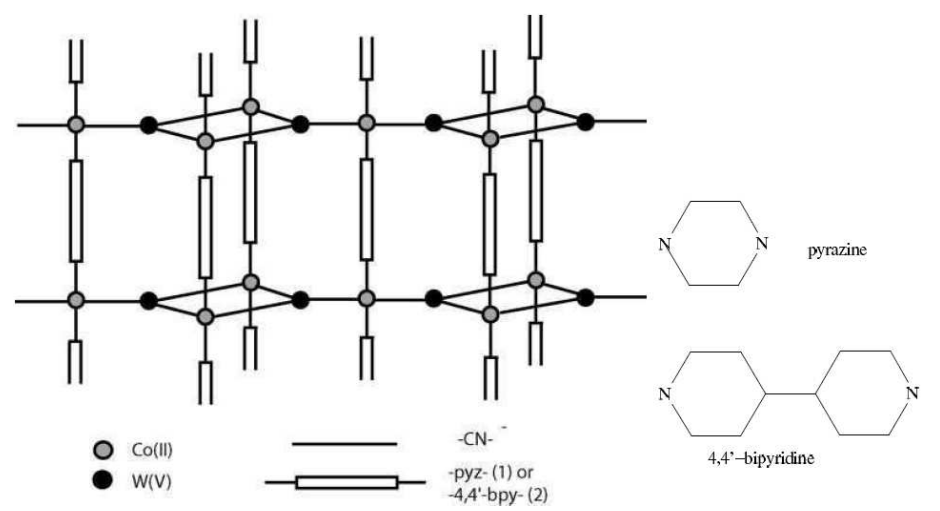

Fig. 1. Schematic view of the $\mathrm{W}-\mathrm{CN}-\mathrm{Co} \mathrm{Co}-\mathrm{bpy}-\mathrm{Co}$ network.

two $\mathrm{N}$ atoms from bpy bridging to the other cobalt and water. The schematic view of the crystal structure is presented in Fig. 1. In our paper we present slow relaxation connected with reorganization of the lattice due to the defects created by light.

\section{Experimental}

The details of the synthesis were described in [7]. Fine powder of deep orange color was obtained, all attempts to obtain single crystal were unsuccessful.

The magnetic measurements were carried out using a 7225 Lake Shore ac susceptometer/dc magnetometer equipped with the probe with an optical fiber. The optical system consists of red $(\lambda=638 \mathrm{~nm})$ and green $(\lambda=532 \mathrm{~nm})$ lasers. The intensity of the light was measured to be $1 \mathrm{~mW} / \mathrm{cm}^{2}$. The details of the system is described elsewhere [8]. Magnetization measurements were performed at helium bath temperature. After cooling the sample to $4.2 \mathrm{~K}$ in zero field, the time dependence of the magnetization for several fixed values of the external field 
was registered. Time step was set to $60 \mathrm{~s}$. After each measurement the sample was heated up to $60 \mathrm{~K}$ and then cooled again to $4.2 \mathrm{~K}$.

\section{Results}

As it was mentioned earlier, our aim was to study long time behavior of magnetization relaxing in the external field. The study of ac susceptibility at different frequencies does not show any changes after few hours of light exposition. The result is presented in Fig. 2. Both real and imaginary parts of ac susceptibility

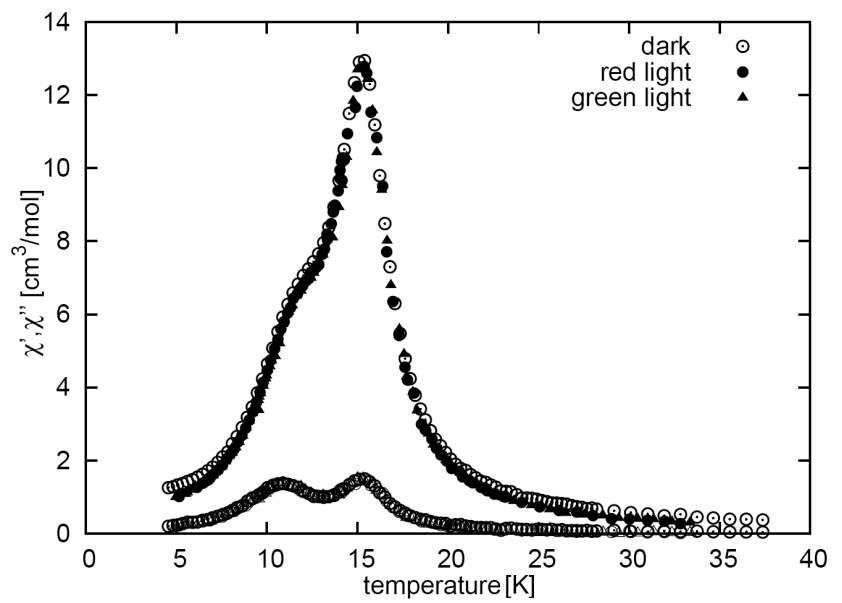

Fig. 2. Real and imaginary ac susceptibility as measured at $125 \mathrm{~Hz}$ and $H_{\mathrm{ac}}=5 \mathrm{Oe}$ measured at dark, green, and red light.

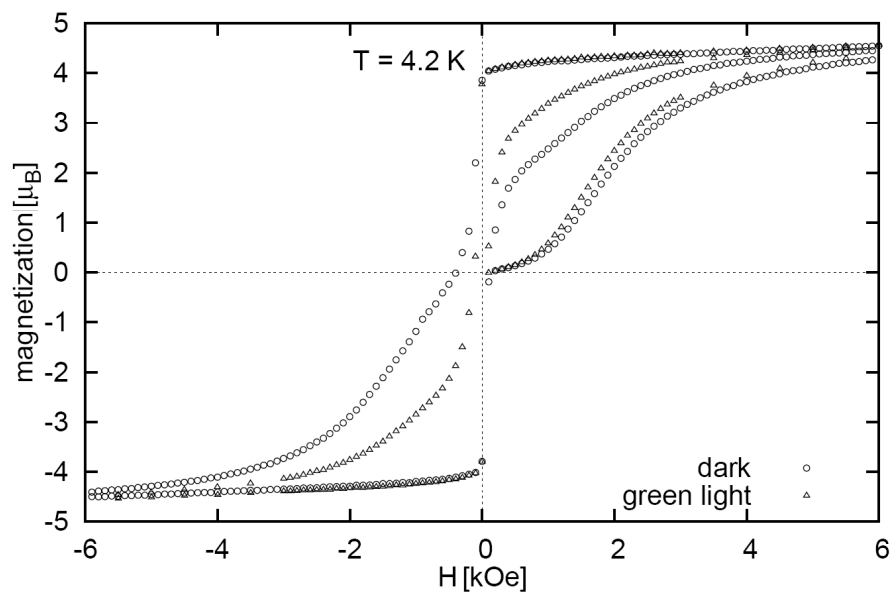

Fig. 3. Magnetic hysteresis measured at $T=4.2 \mathrm{~K}$ at dark and green light. 
indicate magnetic transition at $T_{\mathrm{c}}=16 \mathrm{~K}$ and kind of re-entrant transition at $8 \mathrm{~K}$. After 300 minutes of either red or green light exposure the anomaly does not change. The conclusion is that one needs higher magnetic field in order to observe the effect. In fact the effect is well seen when hysteresis loop is measured as shown in Fig. 3. The virgin curve is about the same in dark as in green light. The fact that the magnetization values in the loop are higher than those of the primary run may indicate that after the saturation in the strong magnetic field only two orientations of the domains are preferred. The most striking effect is seen during long time magnetization measurements and is presented in Fig. 4. When green

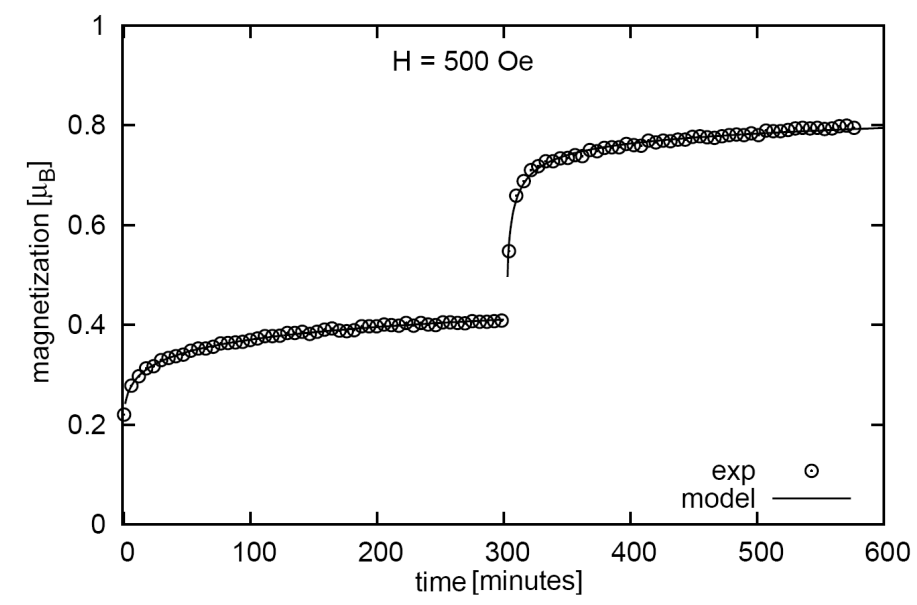

Fig. 4. Magnetization measured at $T=4.2 \mathrm{~K}$ and $H=500$ Oe at dark and green light switched on after 300 minutes. Solid line represents model, open circles are measured values.

light is switched on there is a drastic increase in magnetization rate while under the red light on the effect is very small. When after some time light is switched off the magnetization remains unaltered. It is necessary to heat the sample well above $T_{\mathrm{c}}$ in order to erase the remnant magnetization.

\section{Discussion}

One can expect that the time evolution of magnetization is described by the function usually applied for population dynamics like logistic one

$$
g_{\tau}(t)=\frac{1}{1+\mathrm{e}^{-t / \tau}},
$$

where $\tau$ is characteristic time of evolution. The above equation is the solution of the differential equation

$$
\frac{\mathrm{d} g_{\tau}(t)}{\mathrm{d} t}=\frac{1}{\tau} g_{\tau}(t)\left[1-g_{\tau}(t)\right]
$$

It appears that the time evolution of the magnetization cannot be fitted in the 
wide range of time by one function. It is necessary to use piecewise procedure with many parameters. So we propose the other functional dependence of the magnetization. It is based on the equation of motion

$$
\frac{\mathrm{d} g(t)}{\mathrm{d} t}=-\frac{\beta}{t} g(t)[1-g(t)]
$$

which leads to the function

$$
g(t)=\frac{1}{1+(t / \tau)^{\beta}} .
$$

All numerical fittings were performed for the time dependence of the magnetization given by

$$
M(t)=M(\infty)+[M(0)-M(\infty)] g(t) .
$$

The results of the fitting procedure are shown in Fig. 5. All experimental

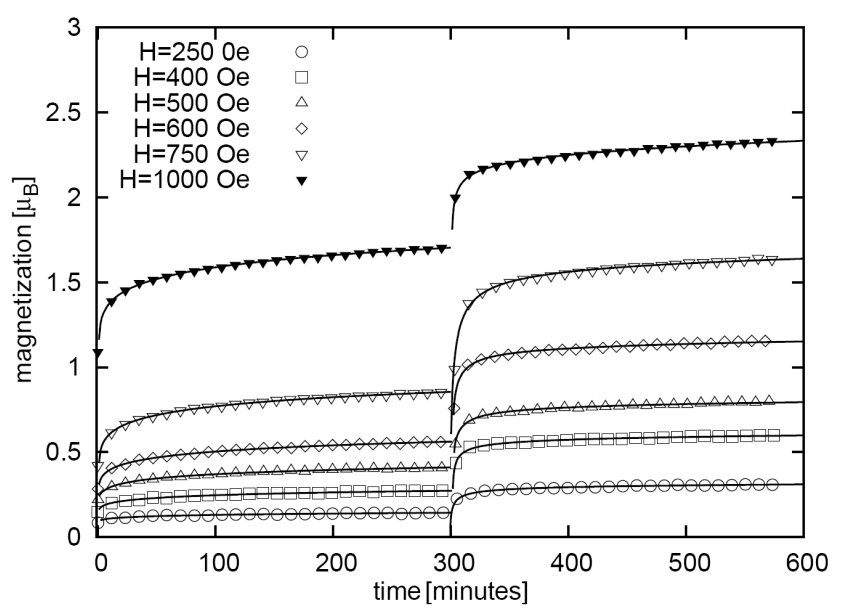

Fig. 5. Time dependence of magnetization at different magnetic fields as measured (points) and model calculation (solid line). After 300 minutes green light was switched on.

points were nicely fitted. The parameters $\tau$ and $\beta$ do not depend on magnetic field within the fitting standard error. There is well seen change in $\tau$ from $300 \pm 20$ minutes in dark to $5 \pm 1$ minutes after irradiation by green light. The parameter $\beta$ changes from 0.3 in dark to 0.7 in green light. The reason for shortening of the relaxation time $\tau$ after light irradiation most probably comes from the lattice rather than molecular origin. The light irradiation produces certain number of defects and so smaller domains can follow magnetic field faster. We propose that the formation of defects corresponds to the inter-valence transfer $\mathrm{Co}^{\mathrm{II}} \mathrm{W}^{\mathrm{V}} \rightleftharpoons \mathrm{Co}^{\mathrm{III}} \mathrm{W}^{\mathrm{IV}}$ process induced by $532 \mathrm{~nm}$ laser line excitation [9]. The defects consist of $\mathrm{Co}(\mathrm{III})\left(d^{6}, S=0\right)$ and $\mathrm{W}(\mathrm{IV})\left(d^{2}, S=0\right)$ metal sites. Thus the path of the magnetic interaction along the chain is broken. Such a diamagnetic defect may speed up the reorientation of the whole domain with respect to the ex- 
ternal field. We have performed similar experiments on the compound consisting of pyrazine instead of bipyridine and we observed smaller effect. One of the reason could be smaller distance between $\mathrm{Co}-\mathrm{W}-\mathrm{Co}$ chains and larger interchain interaction $\left(T_{\mathrm{c}}=26 \mathrm{~K}\right)$. The complicated mechanism of photo-magnetization process of $\mathrm{Co}_{3}^{\mathrm{II}}\left(\mathrm{H}_{2} \mathrm{O}\right)_{4}\left(4,4^{\prime} \text {-bpy }\right)_{3}\left[\mathrm{~W}^{\mathrm{V}}(\mathrm{CN})_{8}\right]_{2} \cdot 1.5\left(4,4^{\prime}\right.$-bpy $) \cdot 6 \mathrm{H}_{2} \mathrm{O}$ requires further detailed studies being currently underway.

\section{Acknowledgments}

This work was partially supported by Network of Excellence MAGMANet (NMP3-CT-2005-515767).

\section{References}

[1] Y. Nakazawa, M. Tamura, N. Shirakawa, D. Shiomi, M. Takahashi, M. Kinoshita, M. Ishikawa, Phys. Rev. B 46, 8906 (1992).

[2] S. Ferlay, T. Mallah, R. Ouahes, P. Vallet, M. Verdaguer, Nature 378, 701 (1995).

[3] R. Sessoli, D. Gatteschi, A. Caneshi, M.A. Novak, Nature 365, 141 (1993).

[4] O. Sato, T. Iyoda, A. Fujishima, K. Hashimoto, Science 272, 704 (1996).

[5] K. Hashimoto, S. Ohkoshi, Philos. Trans. R. Soc. Lond. A 357, 2977 (1999).

[6] M. Nishino, K. Yamaguchi, S. Miyashita, Phys. Rev. B 58, 9303 (1998).

[7] R. Podgajny, M. Bałanda, M. Sikora, M. Borowiec, L. Spałek, C. Kapusta, B. Sieklucka, Dalton Trans., 2801 (2006).

[8] P.M. Zieliński, M. Bałanda, R. Pełka, T. Wasiutyński, J. Phys., Conf. Series 21, 237 (2005).

[9] S. Ohkoshi, S. Ikeda, T. Hozumi, T. Kashiwagi, K. Hashimoto, J. Am. Chem. Soc. 128, 5321 (2006). 